ARTIGOS

\section{FATORES CONDICIONANTES DA SATISFAÇÃO GERAL DOS USUÁRIOS DOS SERVIÇOS DE UMA BIBLIOTECA}

\section{RESUMO}

O objetivo deste artigo é analisar os condicionantes da satisfação dos usuários de uma biblioteca instalada em Instituição de Ensino Superior e testar um modelo de satisfação geral dos usuários dos serviços. Os dados obtidos a partir de um questionário aplicado junto a uma amostra composta por estudantes de uma faculdade foram analisados por meio de regressão linear múltipla. Os resultados evidenciaram que os fatores "espaço físico", "variedade de livros" e "serviços web" estão positivamente relacionados com o nível de satisfação geral indicado pelos estudantes (enquanto usuários dos serviços). Estes resultados estão consoantes com estudos prévios e evidenciam a natureza multidimensional do construto satisfação. As implicações para os gestores referem-se à utilização desses parâmetros e condicionantes como possíveis fatores que podem ser observados na gestão da satisfação dos serviços de bibliotecas. Nesse sentido, com base nos efeitos dos diferentes condicionantes, é possível orientar os esforços de melhoria dos serviços. Parece razoável haver relevância deste artigo, pois há poucos estudos que tratam desta temática, e são raros no contexto estudado.

Palavras-chave: Satisfação. Qualidade dos Serviços. Biblioteca. Marketing.

\section{INTRODUÇÃO}

O setor de ensino pode ser pensado sob a perspectiva do marketing. Para Porto e Réginer (2003), a educação superior deixou de ser sinônimo de um conjunto de instituições, para se tornar um setor em expansão, uma área que atrai recursos e gera oportunidades. Nessa perspectiva, apresentam-se as instituições de ensino superior como provedoras de múltiplos serviços especializados, perpassando várias áreas de especialização técnica e, em alguns casos, envolvendo todas as áreas do conhecimento.

No contexto da prestação de serviços educacionais, a infraestrutura e os serviços disponibilizados como suporte ao processo de ensino têm sido considerados fundamentais. Neste estudo, enfatizaram-se os serviços das bibliotecas, local de recolha e organização de livros e de outros documentos para consulta de públicos 
distintos. A biblioteca propicia aos discentes e docentes o acesso aos materiais e recursos informacionais necessários à criação e à disseminação do conhecimento. Huang (2007) ressalta a necessidade de haver um feedback referente aos serviços prestados. Observa-se, portanto a necessidade de interligação conceitual prática.

Algumas instituições de ensino superior no Brasil carecem de gestores com formação técnica e conhecimento das técnicas específicas de gestão de bibliotecas, como é o caso da mensuração da qualidade dos serviços e dos modelos de satisfação do usuário/cliente. Essa carência de formação gerencial específica no país pode refletir na reduzida utilização das práticas de mensuração de desempenho, incluindo o nível de qualidade dos serviços prestados (TACHIZAWA; ANDRADE, 2006).

Estudos anteriores dedicaram-se a avaliar a satisfação de usuários dos serviços de bibliotecas. Conforme ressaltou Hiller (2001), a aplicação de pesquisas junto aos usuários de bibliotecas tem sido prática relativamente comum em muitas bibliotecas de excelência, servindo como ferramenta de mensuração de desempenho. Algumas ferramentas são exemplificadas nos estudos de Van House, Weil e McClure (1990); Moore (1992); Poll e Boekhorst (1998); Ceynowa e Coners (2002). Contudo, há ainda muita diversidade na abordagem e na utilização de indicadores de desempenho (BLIXRUD, 2003; HERGET; HIERL, 2007; BRASIL, 2011). Adicionalmente, uma vez que a dinâmica da prestação de serviços é sempre dependente, em maior ou menor grau, da participação do próprio cliente, as considerações sobre diferentes contextos organizacionais e culturais são relevantes. Nesse sentido, é evidente a necessidade de novos estudos sobre o tema da satisfação dos usuários de bibliotecas, principalmente considerando o contexto brasileiro.

Em essência, esses estudos anteriores parecem enfatizar que a prestação de serviços, dentro de parâmetros de qualidade desejados pelo usuário final, é fundamental para a satisfação dos usuários. Assim, efetivamente, esse tipo de avaliação deve perpassar à compreen- são das variáveis condicionantes da satisfação dos serviços.

O presente artigo analisa os fatores condicionantes da satisfação geral dos usuários de uma biblioteca instalada em uma Instituição de Ensino Superior privada, localizada no sul do Brasil, com o objetivo de explicar a relação entre variáveis condicionantes da satisfação geral dos usuários dos serviços. Para tanto, foram consideradas algumas hipóteses referentes às relações entre diferentes condicionantes e o nível da satisfação com a biblioteca. Os dados foram obtidos a partir da aplicação de um questionário junto a uma amostra de usuários da biblioteca, e a análise compreendeu a utilização de regressão linear múltipla, conforme detalhado na seção de metodologia deste trabalho.

Quanto à organização do artigo, a próxima seção traz uma revisão da literatura relacionada ao tema e apresenta os elementos envolvidos com o problema de pesquisa. A seção 3 detalha os métodos utilizados, enquanto, em seguida, são apresentados e discutidos os resultados e as conclusões provenientes do estudo.

\section{REVISÃO DA LITERATURA E HI- PÓTESES DO TRABALHO}

Segundo Ajibero (1995), uma biblioteca tem papel fundamental em uma instituição de ensino, já que é o local onde as fontes de informação acadêmica são adquiridas, organizadas, processadas, armazenadas e disponibilizadas para atender às necessidades informacionais dos professores, dos pesquisadores, dos estudantes e da comunidade em geral; os quais representam os diferentes públicos (ou usuários) de uma biblioteca.

Filiz (2007) observa que a satisfação do usuário é uma medida importante para a qualidade dos serviços, porém argumenta que a gestão de bibliotecas, sobretudo em países em desenvolvimento, obteve pouca atenção em tempos recentes. A importância da infraestrutura e dos serviços da biblioteca são reconhecidamente fatores relevantes para a competitividade das organizações de ensino, inclusive quando 
analisada sob a perspectiva dos estudantes, segundo identificou Linhares (2015).

A satisfação do consumidor tem sido um conceito-chave para o marketing; E a complexidade do construto é evidenciada, já que tem recebido várias definições (FORNELL; RUST; DEKIMPE, 2010; RUST; ZAHORIK, 1993). Em termos gerais, compreende um julgamento avaliativo posterior ao consumo, ou seja, depois da experiência com o produto e, ou serviço. Nesse sentido, pode ser pensada com uma reação emocional avaliativa decorrente da experiência de consumo (OLIVER, 1980). O tema tem sido recorrente na pesquisa científica e na pauta de organizações com ou sem fins lucrativos (FORNELL et al., 2006).

Considerando o enfoque da Gestão de Serviços, uma biblioteca centrada na necessidade dos clientes deve identificar claramente seus objetivos, as necessidades particulares dos usuários dos serviços, e desenvolver, com base nessas necessidades, uma oferta de produtos e serviços adequada ao público-alvo. Desse modo, a biblioteca pode ser pensada como parte de uma instituição de ensino superior orientada para o mercado, na qual todas as operações, inclusive aquisição, processamento e armazenamento de informações, são focadas na oferta do serviço. Desse modo, estaria em sintonia com a perspectiva dos seus usuários, buscando responder, de forma eficiente, à demanda existente (KAVULYA, 2004). Assim, a satisfação do usuário pode ser pensada como relevante, no cenário futuro de desenvolvimento de bibliotecas, alguns estudos versam sobre estes fatores, como discorre Inayatullah (2007).

Além disso, a satisfação tem sido um tema recorrente na pauta das organizações interessadas em manter a fidelidade dos usuários (ou clientes) em um mercado competitivo, principalmente pelo fato de melhorar a qualidade dos serviços e produtos de uma organização (FORNELL; RUST; DEKIMPE, 2010; RUST; ZAHORIK, 1993). A literatura indica que os estudos de satisfação dos usuários de bibliotecas utilizam-se de conjuntos de atributos para tentar medir a percepção dos usuários acerca de elementos-chave dos serviços prestados. Algumas pesquisas têm sido utilizadas para avaliar os serviços das bibliotecas (KIRAN, 2010); alguns itens como os procedimentos de empréstimo, as instalações, os serviços e a qualidade das coleções também têm sido avaliados (CEYNOWA; CONERS, 2002).

A ideia é que as análises constituam-se como importante ferramenta de apoio para a tomada de decisões dos gestores e orientem os esforços para que as ações correspondentes possam ser direcionadas, os aspectos de satisfação mantidos e os aspectos de insatisfação corrigidos (KIRAN, 2010). As pesquisas de satisfação, portanto, contribuem para orientar a ação dos gestores e bibliotecários de instituições de ensino.

Referente à associação entre satisfação e qualidade dos serviços, a temática já foi estudada em vários contextos (BEZERRA; GOMES, 2015). De acordo com Filiz (2007), os conceitos de qualidade e satisfação são utilizados conjuntamente e até de forma intercambiável. De acordo com Parasuraman, Zeithaml e Berry (1988), a percepção de qualidade está relacionada com a percepção de superioridade do serviço. Para Lovelock e Wright (2001), a qualidade do serviço é o grau em que o serviço atende ou supera as expectativas do cliente. Assim, se os clientes avaliam entrega do serviço, e prestação do serviço, percebendo como melhor que o esperado, ficam satisfeitos; do contrário, insatisfeitos. Em contextos específicos, como é o caso de mensuração da qualidade dos serviços e satisfação com os serviços em bibliotecas, os estudos parecem um pouco mais raros.

Reafirmando esta perspectiva analítica, Filiz (2007) observa que eventos de satisfação com o serviço geraram, ao longo do tempo, a percepção de qualidade. Nesta mesma perspectiva, Kavulya (2004) explica que os usuários medem regularmente a utilidade de uma biblioteca pelas experiências passadas (eventos) e estabelecem uma consciência de qualidade sobre os tipos de serviços ofertados, comparando com suas expectativas.

Em um estudo que investigou a qualida- 
de dos serviços em bibliotecas de universidades, por meio dos itens constantes no modelo SERVQUAL, cujo objetivo foi quantificar a relação entre a variável dependente e as independentes, Filiz (2007) identificou alguns fatores (qualidade do serviço de biblioteca; qualidade das informações; o ambiente da biblioteca; confiabilidade; qualidade do sistema de catálogo on-line e confiança) que resultaram em bom ajustamento do modelo estudado. $\mathrm{O}$ autor concluiu que estes fatores são importantes para compreender o grau de satisfação dos clientes, usuários, das bibliotecas.

Consoante com a visão de Zeithaml e Bitner (2003), um dos componentes do composto de marketing para serviços são as evidências físicas. Esse componente refere-se ao ambiente no qual o serviço é executado, ao local e ambientação onde a empresa interage com o cliente, assim como a qualquer componente tangível que facilite o desempenho ou a comunicação do serviço. No contexto do serviço de uma biblioteca, materiais impressos, livros, mobiliário e instalações estão nesta categoria. Não obstante à tendência pela virtualização dos serviços, há ainda uma forte relação de significado da biblioteca como um local físico, o que leva à impressão de que os serviços úteis de biblioteca são normalmente presenciais (CHOY, 2011). Portanto, assume-se como hipótese que a percepção do usuário sobre a qualidade do espaço físico esteja relacionada diretamente com sua satisfação geral quanto aos serviços da biblioteca, embora os serviços virtuais também tenham crescido na importância assumida pela preferência do consumidor, considerando os aspectos de simultaneidade desses dois atributos no modelo proposto, e como se comportam.

Referindo-se ao insumo clássico de uma biblioteca, os livros, considera-se que a satisfação do usuário dos serviços da biblioteca muito provavelmente ainda é dependente da qualidade e quantidade desse material. A evidência física, a necessidade de manipulação dos livros, o acervo físico etc., estaria associado à dinâmica do serviço, de modo geral (NITECKI, 1996; HOUSEWRIGHT; SCHONFELD, 2008). As- sim, considera-se como hipótese que a variedade dos livros disponibilizados está diretamente relacionada com a satisfação dos usuários.

Embora o espaço físico, as instalações e mesmo o acervo físico em livros e outras publicações ainda se constituam partes importantes para a satisfação do usuário dos serviços de uma biblioteca, há uma tendência de evolução para outro paradigma, que compreende a demanda por conteúdo e serviços via web, e virtualização do acervo (HOUSEWRIGHT; SCHONFELD, 2008). Parece, realmente, haver uma tendência crescente à virtualização dos serviços e acervo; Com a propagação do uso da internet e a consequente ubiquidade dos conteúdos, em que o som, a imagem e os textos viajam pelo mundo. Todos esses elementos acabam permeando a oferta de materiais. Desse modo, há influências quanto à conveniência e às facilidades nas questões logísticas, uma vez que a informação está convenientemente disponibilizada on-line (CHOY, 2011). Não por acaso, os serviços, possíveis de serem virtualizados, tendem a ser cada vez mais disponibilizados por meio de plataformas web.

Sabe-se que é possível obter acesso às bibliotecas on-line, com disponibilização de obras relevantes para a comunidade acadêmica. Além disso, os serviços das bibliotecas em plataformas on-line estão crescendo e já é possível, em muitos dos casos, acessar e ler parte do acervo, reservar obras, renovar empréstimos de livros físicos e muitos outros serviços com o uso da Internet. Por isso, os serviços web das bibliotecas configuram-se elementos importantes para o nível de satisfação com os serviços de uma biblioteca. Assim, uma terceira hipótese é sugerida: a qualidade dos serviços web está diretamente relacionada à satisfação geral do usuário com a biblioteca.

A partir da contextualização realizada, são sumarizadas as seguintes hipóteses de trabalho, as quais irão compor o modelo teórico de relações entre construtos intrínsecos à satisfação geral dos usuários dos serviços da biblioteca:

a) hipótese 1: A percepção da qualidade 
quanto ao espaço físico está positivamente relacionada com a satisfação geral quanto aos serviços da biblioteca;

b) hipótese 2: A percepção da qualidade quanto à variedade de livros está positivamente relacionada com a satisfação geral quanto aos serviços da biblioteca;

c) hipótese 3: A percepção da qualidade quanto aos serviços web está positivamente relacionada com a satisfação geral quanto aos serviços da biblioteca.

Com relação aos sinais esperados dos coeficientes, espera-se que as variáveis independentes (variedade de livros, serviços web e espaço físico) estejam positivamente relacionadas com a variável dependente (satisfação geral com os serviços da biblioteca) (Figura 1).

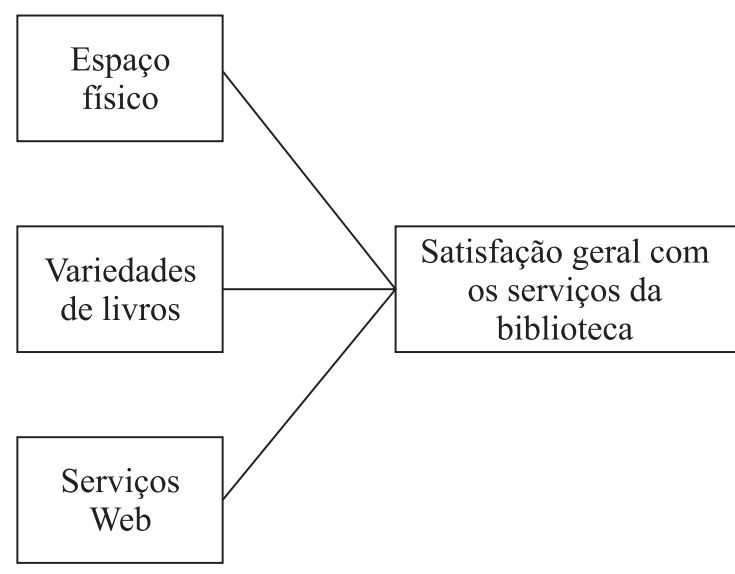

Figura 1 - Modelo teórico

Fonte: elaborada pelos autores deste trabalho com base na pesquisa.

\section{DESENHO METODOLÓGICO}

Este estudo desenvolveu-se conforme as seguintes etapas: (i) coleta de dados; (ii) seleção das variáveis, possíveis condicionantes da satisfação geral com a biblioteca; (iii) estimação do modelo de regressão pelo método dos mínimos quadrados (regressão linear múltipla); (iv) interpretação dos coeficientes; (v) análises estatísticas, teste da multicolinearidade e heteroscedasticidade; (vi) discussões dos resultados.

As variáveis foram selecionadas com o objetivo de avaliar a satisfação geral atinente aos serviços prestados por bibliotecas. Para a coleta de dados, foi utilizado um questionário aplicado aos estudantes de uma faculdade (caracterizada na seção dos resultados) usuários dos serviços da biblioteca. A amostragem compreendeu todos os usuários do período de volta às aulas, pós-férias de julho, aproveitando o instrumento avaliativo da faculdade, e obteve 539 respondentes e 536 questionários válidos, sendo excluídos três $\left(\mathrm{n}_{\mathrm{s}}\right)$ por motivo dos dados incompletos.

$\mathrm{O}$ questionário foi do tipo estruturado e compreendeu somente questões objetivas, por meio das quais os respondentes foram solicitados a indicar suas opiniões sobre cada item, conforme uma escala likert de cinco pontos, sendo o número 1 equivalente ao mais baixo nível de satisfação e 5, ao mais alto nível de satisfação. $O$ questionário foi anteriormente testado, para evitar o uso de linguagem inadequada e minimizar a subjetividade, de modo que as respostas provavelmente não foram condicionadas pela própria estrutura e estrutura da linguagem utilizada no questionário, havendo compreensão das perguntas por parte dos respondentes.

As análises estatísticas foram realizadas utilizando-se do sistema Gretl, com uso da técnica de regressão linear múltipla. O Gretl é um software para análises estatísticas com acesso livre, o que provê uma diversidade de técnicas estatísticas. Uma das principais qualidades da ferramenta é a simplicidade de uso e a possibilidade de realização de vários testes que atendem aos pressupostos da regressão linear múltipla. O tratamento estatístico foi orientado pelas referências indicadas pela literatura especializada (GUJARATI; PORTER, 2011). O modelo linear foi estimado segundo a formulação seguidamente apresentada, considerando que as análises e seus respectivos coeficientes foram obtidos pelo método dos mínimos quadrados.

$$
Y_{i}=\beta_{0}+\beta_{I} X_{1}+\beta_{2} X_{2}+\beta_{3} X_{3}+\mu_{i}
$$

As variáveis utilizadas são descritas a seguir. 
a) Variável Dependente

$\mathrm{Y}=$ Nível de satisfação geral com os serviços da biblioteca

b) Variáveis Independentes

$\mathrm{B}_{0}=$ Corresponde a variável constante do modelo.

$\mathrm{X}_{1}=$ Percepção relativa à satisfação com o espaço físico da biblioteca.

$\mathrm{X}_{2}=$ Percepção relativa à satisfação com os serviços web prestados pela biblioteca.

$\mathrm{X}_{3}=$ Percepção relativa à satisfação com a variedade de livros da biblioteca.

As variáveis independentes referem-se à percepção dos usuários dos serviços da biblioteca, relativa à satisfação medida em uma escala Likert, refletindo o nível de satisfação em uma escala de 5 pontos.

\section{RESULTADOS}

Esta seção apresenta, primeiramente, uma breve descrição da instituição foco da pesquisa e o contexto organizacional. Em seguida, são apresentados os coeficientes estimados e o resultado do teste das hipóteses.

A biblioteca está instalada em uma instituição de ensino superior privada (nome não identificado para preservar a confidencialidade dos dados), sediada no Estado do Rio Grande do Sul, Brasil. Quanto ao contexto socioeconômico da cidade, a faculdade, encontra-se em um bem desenvolvido parque industrial de alta competi- tividade, que tem apresentado permanentemente superávit em sua relação de negócios com o mercado externo, colocando produtos em diversos países. Além deste setor, há uma variedade de empresas do setor de prestação de serviços, o que permite comparar, em termos relativos, com as grandes metrópoles brasileiras.

Considerando o crescimento e desenvolvimento nesses setores, é crescente a demanda por profissionais capacitados que dominem a prática de conhecimentos técnico-científicos, para atender às demandas das organizações. Neste sentido, a proposta pedagógica da faculdade está direcionada ao atendimento das lacunas de formação no mercado regional. Dessa forma, a qualificação dos espaços formativos é essencial.

Atinente às questões metodológicas do estudo, e seus resultados, a técnica estatística utilizada para as análises foi a regressão linear múltipla. Assim, a satisfação geral com os serviços da biblioteca foi definida como variável dependente, e as seguintes variáveis independentes ou explicativas foram consideradas: (i) espaço físico; (ii) serviços web; (iii) variedade de livros.

As três variáveis explicativas testadas apresentaram significância estatística $(\mathrm{p}<0,05)$ na definição da satisfação geral com os serviços da biblioteca, indicando uma relação estatisticamente significativa entre a satisfação e os fatores estudados. Os coeficientes, o erro padrão, o valor da estatística t, e a significância estatística estão sumarizados na tabela que segue:

Tabela 1 - Resultados do teste estatístico

\begin{tabular}{l|c|c|c|c}
\hline \multicolumn{1}{c|}{ Variável } & Coeficiente & Erro Padrão & Estatística t & Valor p \\
\hline Constante & 0,474505 & 0,138006 & 3,438 & 0,00063 \\
\hline Espaço físico & 0,213991 & 0,0428636 & 4,992 & $<0,00001$ \\
\hline Serviços web & 0,153886 & 0,0352240 & 4,369 & 0,00002 \\
\hline Variedades de livros & 0,251451 & 0,0473039 & 5,316 & $<0,00001$ \\
\hline
\end{tabular}

Nota: A média da variável dependente foi de $=2,14498$, e seu desvio padrão $=1,23925$. $O \mathrm{R}^{2}$ não ajustado foi $=0,24016$ e o $\mathrm{R}^{2}$ ajustado $=0,23589$.

Fonte: dados da pesquisa.

A partir desses resultados, pode-se dizer que aproximadamente $24 \%$ da variabilidade da satisfação geral do usuário com a biblioteca (variável dependente) é explicada pelas variáveis explicativas. Este indicativo denota à existência de mais variáveis que poderiam explicar 
a variabilidade da satisfação geral. Com relação à avaliação geral do modelo, obteve-se estatística-F $(3,534)=56,2597($ valor $p<0,00001)$, o que leva a concluir que o modelo é significativo ao nível de $1 \%$.

A análise dos resíduos demonstrou que se distribuem segundo o padrão da normalidade. Há uma tendência de homogeneidade na dispersão dos resíduos e poucas observações excederam o erro padrão. $\mathrm{O}$ teste de White para a heterocedasticidade retornou o seguinte resultado: estatística de teste: $\mathrm{TR}^{\wedge} 2=38,751853$, com valor $\mathrm{p}=\mathrm{P}($ Qui-quadrado(9) $>38,751853)=$ 0,000013 . Portanto, conclui-se que não se aceita a hipótese da heteroscedasticidade, pois o valor da estatística está fora da zona de aceitação.

$\mathrm{O}$ teste da multicolinearidade aplicado foi o de Fatores de Inflacionamento da Variância (VIF). O resultado aponta que não há indício de multicolinearidade, pois os valores de VIF são menores que 10 , e a matriz de correlações apresenta valores compatíveis com a independência das variáveis independentes, não havendo alta correlação entre as variáveis independentes.

O modelo estimado apresentou o seguinte resultado:

$Y=0,4745+0,2139 x_{1}+01538 x_{2}+0,2514 x_{3}$

A interpretação do modelo é a seguinte:

$\hat{Y}=$ Variável dependente - corresponde ao nível de satisfação geral com os serviços da biblioteca;

$\vec{B}_{0}=$ Variável constante do modelo - é independente do valor das variáveis independentes, e esta reflete com acréscimo de 0,4745 o nível de satisfação em Y.

$B_{1}^{*}=$ Refere-se à variável espaço físico - cada aumento de uma unidade em $\mathrm{X}_{1}$ no nível de satisfação com o espaço físico, reflete 0,2139 unidade com o aumento positivo no nível de satisfação geral com os serviços da biblioteca, na condição ceteris paribus.
$B_{2}=$ Refere-se à variável serviços Web - cada aumento de uma unidade em $\mathrm{X}_{2}$, no nível de satisfação com os serviços web, reflete aumento de 0,1538 unidade no nível de satisfação geral com os serviços da biblioteca, na condição ceteris paribus.

$B_{3}=$ Refere-se à variável variedade de livros para cada aumento de uma unidade na variável $\mathrm{X}_{3}$, no nível de satisfação com a variedade de livros, reflete 0,2514 unidade, com aumento positivo no nível de satisfação geral com os serviços da biblioteca, na condição ceteris paribus.

Referente às três variáveis independentes analisadas, a "variedade dos livros" é o fator que apresentou maior efeito sobre a satisfação geral com os "serviços da biblioteca", seguida pelo "espaço físico", como segundo fator mais importante, entre os analisados. $\mathrm{O}$ fato de o fator serviços web apresentar-se com menos efeito na satisfação com os serviços da biblioteca pode ser uma evidência de que o elemento físico ainda é um fator importante na satisfação dos usuários da biblioteca. Esta evidência parece evidenciar para a necessidade de sistemas híbridos (INAYATULLAH, 2007), físicos e virtuais.

Os resultados da regressão mostram que as variáveis independentes são condicionantes sobre a satisfação com os serviços da biblioteca. A hipótese 1 , referente à percepção da qualidade relativo ao espaço físico, por estar positivamente relacionada com a satisfação geral dos serviços da biblioteca, foi confirmada, tendo o sinal do coeficiente provendo resultado de acordo com o previsto e a relação estatística significativa. Este resultado apresenta-se consoante com os estudos de Inayatullah (2007), já que o espaço físico é um elemento a ser considerado como importante em uma biblioteca, assim como o ambiente virtual.

Referente à hipótese 2, pressupõe que a percepção da qualidade, referente à variedade de livros, está positivamente relacionada com a satisfação geral com os serviços da biblioteca; o sinal do coeficiente é positivo, conforme 
o esperado, e mostrou-se com significância estatística. Este resultado provavelmente seja justificado pelo fato de que a variedade de livros propicia uma maior exposição a diferentes materiais, permitindo ao professor e ao aluno acesso a maior número de estudos.

Referente à hipótese 3, pressupõe que a percepção da qualidade, referente aos serviços web, está positivamente relacionada com a satisfação geral dos serviços da biblioteca, obteve o sinal do coeficiente conforme o esperado, e é significativa, o que indica, nesta amostra, que a satisfação dos usuários da biblioteca é condicionada também pelos serviços web. Isso ressalta, no sentido da tendência, uma maior oferta de serviços web em bibliotecas, conforme a literatura indica (HOUSEWRIGHT; SCHONFELD, 2008).

\section{CONSIDERAÇÕES FINAIS}

O presente estudo teve como objetivo examinar os fatores associados à satisfação geral com os serviços de biblioteca em uma amostra de estudantes de uma faculdade. Os resultados evidenciaram que os três fatores considerados, (i) o espaço físico; (ii) a variedade de livros e (iii) os serviços web são significativos e estão positivamente relacionados com a satisfação geral dos estudantes, diante dos serviços da biblioteca.

Esses resultados convergem com as proposições de estudos anteriores sobre a satisfação dos usuários de bibliotecas com os serviços. Segundo Inayatullah (2007), especificamente ao contexto de bibliotecas, ambientes híbridos, que compreendem tanto o meio virtual quanto os recursos físicos, são importantes componentes na oferta dos serviços. Já Zeithaml e Bietner (2003), na análise do composto de marketing em serviços, evidenciam que ambos, os bens tangíveis e intangíveis, são necessários, e a evidência física é um dos componentes relatados nessa abordagem teórica, enquanto elemento tangível.

A relação significativa dos três fatores é coincidente também com os resultados de Filiz (2007), que observou a importância do acervo on- -line e do ambiente físico em bibliotecas. A ideia e o formato da biblioteca enquanto um local físico não podem ser descartados (CHOY, 2011), embora pareça haver uma tendência contrária (HOUSEWRIGHTE; SCHONFELD, 2008). Além disso, o presente estudo corrobora com uma evidência que converge com a idéia dos espaços híbridos, com serviços via plataforma web, e também com instalações e acervo físico compatível com as expectativas de seus públicos.

Esses modelos híbridos para bibliotecas devem considerar tantos aspectos físicos como equipamentos, instalações, livros e aspectos intangíveis, no geral disponibilizados em plataformas web. Os e-books, os materiais digitalizados, os conteúdos digitais em geral (áudio e vídeo disponibilizados em acervos digitais), além de revistas eletrônicas e bases de dados, e novos adventos tecnológico-digitais devem ser considerados e avaliados quanto à sua disponibilidade e enquanto fatores que podem influenciar a satisfação geral com os serviços de uma biblioteca.

As implicações práticas deste estudo supõem que tanto o espaço físico, quanto os serviços web e a variedade de livros devem ser fatores monitorados pelos gestores e bibliotecários de instituições de ensino a fim de maximizar a satisfação dos usuários dos serviços. Para isso, parece importante haver sondagens constantes entre os professores, alunos e público, procurando identificar a variedade de livros necessária. Nesse sentido, também se faz necessário disponibilizar um espaço físico adaptado às expectativas, para que permita amplo acesso aos recursos informacionais e, complementarmente, os serviços virtuais cada vez mais parecem ganhar importância e fazer parte das expectativas referentes à oferta de informação das bibliotecas.

As limitações da pesquisa consideram relativamente limitante a tipologia utilizada, cross section, com questionários aplicados em um único período. Assim, pesquisas longitudinais para acompanhamento da satisfação dos usuários ao longo do tempo parecem necessárias. Outra limitação refere-se à reduzida abrangência desta investigação, já que foi realizada em uma úni- 
ca instituição de ensino superior. A aplicação da pesquisa em outras instituições, em diferentes regiões do país e em âmbito internacional, inclusive, pode permitir uma visão mais abrangente dos fatores responsáveis pela satisfação dos usuários das bibliotecas. Afinal, há de se considerar também possíveis diferenças culturais e realidades contextuais distintas. Ainda neste aspecto, ressalta-se que ampliar o espectro dos públicos pode permitir resultados distintos.

Novos estudos poderiam identificar outros fatores de forma exploratória, em um primeiro momento, buscando identificar novos fatores, e, posteriormente, testar um modelo mais amplo. Estudos que analisem as questões culturais envolvidas, em em diferentes espaços geográfico culturais, além de públicos distintos, poderiam permitir a evolução do conhecimento neste campo de estudo. Ainda, a ampliação do tempo de coleta de dados (pesquisas longitudinais), além da expansão do número de bibliotecas pesquisadas poderiam trazer possibilidades de comparação com estes resultados, e, novos fatores poderiam possibilitar compreender melhor os condicionantes da satisfação dos usuários dos serviços de bibliotecas, pois as bibliotecas, parecem passar por mudanças fundamentais nos serviços, design e propósito, principalmente considerando as questões associadas à tecnologia.

\section{CONDITIONAL FACTORS OF USER SATISFACTION OF LIBRARY SERVICES}

\begin{abstract}
The objective of this study is to analyze the conditional factors of user satisfaction within a library in a university and to test a model of general user satisfaction with the services provided. The data was collected from a survey applied to a sample of students, and data analysis comprised multiple regression analysis. The results showed that factors "physical space", "variety of books" and "web services" are positively related to the overall level of satisfaction presented by the students as users of library services. These
\end{abstract}

findings are consistent with previous studies and highlight the multidimensional nature of the construction of satisfaction. Regarding the implications for managers, these different factors should be considered as important parameters to be observed in the analysis of customer satisfaction of library services. In this respect, based on the effects of these different determinants of customer satisfaction, it is possible to direct the efforts for the improvement of the quality of the services provided. The article seems to be relevant, as there are only a few studies that deal with this subject - and they are especially rare in the aforementioned context.

Keywords: Satisfaction. Quality service. Library. Marketing.

\section{FACTORES CONDICIONANTES EN LA SATISFACCIÓN GENERAL DE LOS USUARIOS DE SERVICIOS DE UNA BIBLIOTECA}

\section{RESUMEN}

El objetivo del artículo es analizar los condicionantes de la satisfacción de los usuarios de una biblioteca ubicada en una institución de enseñanza superior y testar un modelo de satisfacción general de los usuarios de los servicios. Los datos obtenidos a partir de un cuestionario aplicado a un grupo de estudiantes de una facultad fueron analizados por medio de regresión linear múltiple. Los resultados evidenciaron que los factores "espacio físico", "variedad de libros" y "servicios web" están positivamente relacionados con el nivel de satisfacción general indicado por los estudiantes que utilizan los servicios. Estos resultados están consonantes con estudios previos y evidencian la naturaleza multidimensional de la construcción de la satisfacción. Las implicaciones para los gestores se refieren a la utilización de estos parámetros y condicionantes como posibles factores que pueden ser observados en la gestión de la satisfacción de los servicios de bibliotecas. En ese sentido, con base en los efectos de las diferentes condicionantes, es 
posible orientar los esfuerzos de mejoramiento de los servicios. Parece razonable haber relevancia de este artículo porque hay muy pocos estudios que tratan de esta temática - y aún más raros en el contexto estudiado.

Palabras-clave: Satisfacción. Cualidad de los servicios. Biblioteca. Marketing.

\section{FACTEURS DETERMINANTS DE LA SATISFACTION GENERAL DES UTILISATEURS DES SERVICES D'UNE BIBLIOTHEQUE}

\section{RESUME}

L'objectif de l'article est d'analyser les déterminants de la satisfaction des utilisateurs d'une bibliothèque installée dans une institution d'enseignement supérieur et de tester un modèle de satisfaction générale des utilisateurs des services. Les données obtenues à partir d'un questionnaire appliqué à un échantillon composé par des étudiants d'une faculté ont été analysés à travers de regression linéaire multiple. Les résultats ont mis en évidence que les facteurs « espace physique », « variété de libres » et « services web » sont positivement liés avec le niveau de satisfaction générale indiquée par les étudiants en tant qu'utilisateurs des services). Ces résultats sont en consonnance avec des études antérieures et mettent en évidence la nature multidimensionnelle de la construction de la satisfaction. Les implications pour les gérants se réfèrent à l'utilisation de ces paramètres et déterminants comme facteurs qui peuvent observés dans la gestion de la satisfaction des services de bibliothèques. Dans ce sens, sur la base des effets des différents déterminants, il est possible d'orienter les efforts d'amélioration des services. Il semble raisonable que l'article soit pertinent, puisqu'il $\mathrm{y}$ a très peux d'études qui traitent de cette thématique - et qu'ils sont encore plus rare dans le contexte etudié.

Mots-clés: Satisfaction. Qualité des services. Bibliothèques. Marketing.

\section{REFERÊNCIAS}

AJIBERO, M. I. Users expectations from Nigerian universities library services in the $21 \mathrm{st}$ century. Quarterly, v. 1, n.1, p. 33-49, 1995.

BEZERRA, G. C. L.; GOMES, C. F. The effects of service quality dimensions and passenger characteristics on passenger's overall satisfaction with an airport. Journal of Air Transport Management, United Kingdom, v. 44-45, p. 77-81, 2015.

BLIXRUD, J. C. Assessing library performance: new measures, methods, and models. In: IATUL CONFERENCE., 24., 2003, Ankara. Anais... Ankara: Purdue University, 2003.

BRASIL, H. S. Avaliação do processo de modernização da biblioteca da universidade federal do Tocantins. 2011. 184 f. Dissertação (Mestrado em Avaliação de Políticas Públicas) - Universidade Federal do Ceará, Fortaleza, Ceará, Brasil, 2011.

CEYNOWA, K.; CONERS, A. Balanced scorecard für Wissenschaftliche Bibliotheken. Frankfurt: ZfBB Sonderheft, Klostermann, 2002.

CHOY, F. C. From library stacks to library-ina-pocket: will users be around? Library Management, United Kingdom, v. 32, n. 1/2, p. 62$72,2011$.

FILIZ, Z. Service quality of university library: a survey amongst students at Osmangazi University and Anadolu University. Istanbul University Econometrics and Statistics e-Journal, v. 4, n. 1, p. 1-19, 2007.

FORNELL, C. et al. Customer satisfaction and stock prices: high returns and low risk. Journal of Marketing, United States, v.70, n. 1, p.1-14, 2006.

FORNELL, C.; RUST, R. T.; DEKIMPE, M. $\mathrm{G}$. The effect of customer satisfaction on con- 
sumer spending growth. Journal of Marketing Research, United States, v. 47, n. 1, p. 2835, 2010 .

GUJARATI, D. N.; PORTER, D. C. Econometria básica. 5. ed. Porto Alegre: Bookman, 2011.

HERGET, J.; HIERL, S. Excellence in libraries: a systematic and integrated approach. New Library World, United Kingdom, v. 108, n. 11/12, p.526-544, 2007.

HILLER, S. Assessing user needs, satisfaction, and library performance at the University of Washington libraries. Library Trends, United States, v. 49, n. 4, p. 605-625, 2001.

HOUSEWRIGHT, R.; SCHONFELD, R. Ithaka's 2006 studies of key stakeholders in the digital transformation in higher education. 2008. Disponível em: $<$ http://librariesofthefuture.jiscinvolve.org/wp/files/2009/03/ithaka_ review_v1-8.doc $>$. Acesso em: 15 set. 2013.

HUANG, R. Study and analysis of information on the reader's potential discontent in an academic library. Library Management, United Kingdom, v. 28, p. 27-35, 2007.

INAYATULLAH, S. Which future for libraries? Foresight, United Kingdom, v. 9, n. 3, p. 54-57, 2007.

KAVULYA, J. M. Marketing of library services: a case study of selected university libraries in Kenya. Library Management, United Kingdom, v. 25, n. 3, p. 118-126, 2004.

KIRAN, K. Service quality and customer satisfaction in academic libraries: perspectives from a Malaysian university. Library Review, United Kingdom, v. 59, n. 4, p. 261-273, 2010.

LINHARES, T. A. S Fatores críticos de sucesso nas instituições de ensino superior privadas no Distrito Federal - Brasil. 2015. 136 f.
Dissertação (Mestrado em Gestão da Formação e Administração Educacional) - Universidade de Coimbra, Coimbra, 2015.

LOVELOCK, C.; WRIGHT, L. Serviços marketing e gestão. São Paulo: Saraiva, 2001.

MOORE, N. Leistungsmessung in öffentlichen bibliotheken. O Praxis, v. 16, n. 2, p. 169-196, 1992.

NITECKI, D. Changing the concept and measure of service quality in academic libraries. Journal of Academic Librarianship, United Kingdom, v. 22, n. 3, p. 181-190, 1996.

OLIVER, R. L. A cognitive model of the antecedents and consequences of satisfaction decisions. Journal of Marketing Research, United States, v. 17, n. 4, p.460-469, 1980.

PARASURAMAN, A.; ZEITHAML, V. A.; BERRY, L. SERVQUAL: a multiple-item scale for measuring consumer e perceptions of service quality. Journal of Retailing, United Kingdom, v. 64, p. 12-40, 1988.

POLL, R.; BOEKHORST, P. Measuring quality: international guidelines for performance measurement in academic libraries. Munchen: IFLA Publications, 1998.

PORTO, C.; RÉGINER, K. O ensino superior no mundo e no Brasil: condicionantes, tendências e cenários para o horizonte 2003-2025: uma abordagem exploratória. Brasília: Macroplan, 2003.

RUST, R.; ZAHORIK, A. Customer satisfaction, customer retention and market share. Journal of retailing, United Kingdm, v. 69. p. 193-215, 1993.

TACHIZAWA, T.; ANDRADE, R. O. B. Gestão de instituições de ensino. 4. ed. Rio de Janeiro: FGV, 2006. 
VAN HOUSE, N. A.; WEIL, B.; MCCLURE, C. R. Measuring academic library performance. Chicago: American Library Association, 1990.

ZEITHAML, A. V.; BITNER, J. M. Marketing de serviços: a empresa com foco no cliente. 2. ed. Porto Alegre: Bookman, 2003. 